\title{
Orthogonal Families of Real Sequences
}

\author{
Arnold W. Miller \\ and \\ Juris Steprans
}

For $x, y \in \mathbb{R}^{\omega}$ define the inner product

$$
(x, y)=\Sigma_{n \in \omega} x(n) y(n)
$$

which may not be finite or even exist. We say that $x$ and $y$ are orthogonal if $(x, y)$ converges and equals 0 .

Define $l_{p}$ to be the set of all $x \in \mathbb{R}^{\omega}$ such that

$$
\sum_{n \in \omega}|x(n)|^{p}<\infty
$$

For Hilbert space, $l_{2}$, any family of pairwise orthogonal sequences must be countable. For a good introduction to Hilbert space, see Retherford [幽.

Theorem 1 There exists a pairwise orthogonal family $F$ of size continuum such that $F$ is a subset of $l_{p}$ for every $p>2$.

It was already known that there exists a family of continuum many pairwise orthogonal elements of $\mathbb{R}^{\omega}$. A family $F \subseteq \mathbb{R}^{\omega} \backslash \mathbf{0}$ of pairwise orthogonal sequences is orthogonally complete or a maximal orthogonal family iff the only element of $\mathbb{R}^{\omega}$ orthogonal to every element of $F$ is $\mathbf{0}$, the constant 0 sequence.

It is somewhat surprising that Kunen's perfect set of orthogonal elements is maximal (a fact first asserted by Abian). MAD families, nonprincipal ultrafilters, and many other such maximal objects cannot be even Borel.

Theorem 2 There exists a perfect maximal orthogonal family of elements of $\mathbb{R}^{\omega}$.

\footnotetext{
${ }^{1}$ Probably Kunen was the first. His example is given in the proof of Theorem 2. Earlier work was done by Abian and examples were also constructed by Keisler and Zapletal independently. At any rate, we know definitely that we didn't do it first.
} 
Abian raised the question of what are the possible cardinalities of maximal orthogonal families.

Theorem 3 In the Cohen real model there is a maximal orthogonal set in $\mathbb{R}^{\omega}$ of cardinality $\omega_{1}$, but there is no maximal orthogonal set of cardinality $\kappa$ with $\omega_{1}<\kappa<\mathfrak{c}$.

By the Cohen real model we mean any model obtained by forcing with finite partial functions from $\gamma$ to 2 , where the ground model satisfies GCH and $\gamma^{\omega}=\gamma$.

Theorem 4 For any countable standard model $M$ of $Z F C$ and $\kappa$ in $M$ such that $M \models \kappa^{\omega}=\kappa$, there exists a ccc generic extension $M[G]$ such that the continuum of $M[G]$ is $\kappa$ and in $M[G]$ for every infinite cardinal $\alpha \leq \kappa$ there is a maximal orthogonal family of cardinality $\alpha$.

Theorem 5 (MA $A_{\kappa}(\sigma$-centered $\left.)\right)$ Suppose $X \subseteq \mathbb{R}^{\omega},\|X\| \leq \kappa, X \cap l_{2}$ is finite, and for every distinct pair $x, y \in X$ the inner product $(x, y)$ converges. Then there exists $a z \in \mathbb{R}^{\omega} \backslash l_{2}$ such that $z$ is orthogonal to every element of $X$.

The question arises of whether uncountable families of pairwise orthogonal elements of $\mathbb{R}^{\omega}$ must somehow determine an almost disjoint family of subsets of $\omega$. In the following result we give a perfect family of orthogonal elements of $\mathbb{R}^{\omega}$ each of which has full support. It is possible to modify Kunen's example, Theorem 2, or the method of Theorem 11 to produce such a perfect set by replacing the zeros by a very small sequence of positive weights. If this' is done, then the resulting elements will be " $l_{2}$ almost disjoint" in the following sense. Given $x$ and $y$ there will be $X$ and $Y$ almost disjoint subsets of $\omega$ such that

$$
\sum_{n \notin X} x(n)^{2}<\infty \text { and } \sum_{n \notin Y} y(n)^{2}<\infty .
$$

${ }^{2}$ Briefly, use the weights $a_{n}=\frac{1}{2^{4 n}}$ off of the comb on level $n$. Use weights $+-b_{n}$ on the comb with $b_{0}$ on the root node, $-b_{n}$ on the teeth, and $+b_{n}$ on the branch. By choosing $b_{n+1}$ so that

$$
b_{0}^{2}+2 b_{1}^{2}+\ldots+2 b_{n}^{2}-2 b_{n+1}^{2}+\sum_{i \leq n+1} a_{i}^{2}\left(2^{i}-2\right)+\sum_{i>n+1} a_{i}^{2}\left(2^{i}-4\right)=0,
$$

the inner products will be zero. 
Equivalently,

$$
\sum_{n \in \omega} \min \{|x(n)|,|y(n)|\}^{2}<\infty
$$

Note that the supports of $x$ and $y$ are almost disjoint iff the function

$$
n \mapsto \min \{|x(n)|,|y(n)|\}
$$

is eventually zero. Consequently, the minimum function is one measure of the almost disjointedness of $x$ and $y$. Note, however that if the inner product of $x$ and $y$ converges, then $\min \{|x(n)|,|y(n)|\} \rightarrow 0$ as $n \rightarrow \infty$.

Theorem 6 There exists a perfect set $P \subseteq \mathbb{R}^{\omega}$ such that every pair of elements of $P$ are orthogonal, $\operatorname{supp}(x)=\omega$ for every $x \in P$, and if we define

$$
h(n)=\min \{|x(n)|: x \in P\}
$$

then for every $p$

$$
\sum_{n<\omega} h(n)^{p}=\infty
$$

K.P.Hart raised the question of whether there could be a maximal orthogonal family in $l_{2}$ which was not maximal in $\mathbb{R}^{\omega}$. This was answered by Kunen and Steprans independently.

Theorem 7 (a) There exists $X$ which is a maximal orthogonal family in $l_{2}$ such that for all $n$ with $1 \leq n \leq \omega$ there exists $Y \subseteq \mathbb{R}^{\omega} \backslash l_{2}$ with $\|Y\|=n$ and $X \cup Y$ a maximal orthogonal family in $\mathbb{R}^{\omega}$. Furthermore, every maximal orthogonal family containing $X$ is countable.

(b) There exists a perfect maximal orthogonal family $P \subseteq \mathbb{R}^{\omega}$ such that $P \cap l_{2}$ is a maximal orthogonal family in $l_{2}$.

The Proofs

\section{Proof of Theorem 1.}

Here is the basic idea. Take the full binary tree and attach pairwise disjoint finite sets $F_{s}$ to each node, see figure 11.

Instead of taking branches, take "combs", which are a branch together with nodes which are just off the branch, e.g. for rightmost branch the comb 

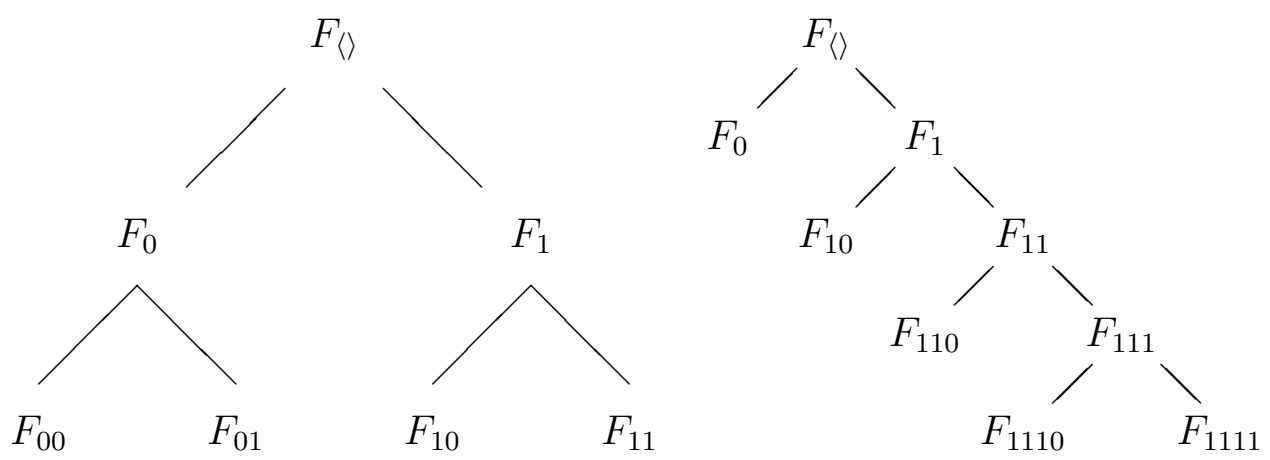

Figure 1: Combs

would be as in figure 1. The elements of the comb will be the support of sequence. Attach to the branch nodes $F_{111 . .1}$ positive weights and to the off branch nodes $F_{111 \ldots 10}$ negative weights. Then when two combs eventually disagree the lowest level pair of nodes gives a negative value. By choosing the sizes of the $F_{s}$ 's and attached weights correctly this negative value will cancel out all the positive values above.

Define the sequence $r_{n}$ by $r_{0}=1$ and

$$
r_{n+1}=\sum_{i \leq n} r_{i} .
$$

(This makes $r_{n}=2^{n-1}$ for $n>0$, but it is irrelevant.) Let $p_{n}>2$ be a sequence decreasing to 2 . Next construct integers $k_{n}>r_{n}$ and reals $\epsilon_{n}>0$ such that

$$
\epsilon_{n}^{2} \cdot k_{n}=r_{n}
$$

and

$$
\epsilon_{n}^{p_{n}} \cdot k_{n} \leq \frac{1}{n^{2}} .
$$

To do this first pick $k_{n}$ so that

$$
k_{n}^{\frac{p_{n}}{2}-1} \geq n^{2} \cdot r_{n}^{\frac{p_{n}}{2}}
$$

then let

$$
\epsilon_{n}^{2}=\frac{r_{n}}{k_{n}} .
$$


Thus

$$
\epsilon_{n}^{p_{n}} \cdot k_{n}=\left(\frac{r_{n}}{k_{n}}\right)^{\frac{p_{n}}{2}} \cdot k_{n}=\frac{r_{n}^{p_{n} / 2}}{k_{n}^{p_{n} / 2-1}} \leq \frac{1}{n^{2}} .
$$

Let $2^{\omega}$ be the set of infinite sequences of 0 and 1 's and let $2^{<\omega}$ be the set of finite sequences of 0 and 1's. Let $\left\{F_{s}: s \in 2^{<\omega}\right\}$ be pairwise disjoint subsets of $\omega$ such that $\left\|F_{s}\right\|=k_{n}$ when $s \in 2^{n}$.

For $x \in 2^{\omega}$ and $n \in \omega$ define

$$
s_{n+1}^{x}=\left.x\right|_{n+1}
$$

and

$$
t_{n+1}^{x}=\left.x\right|_{n} \wedge(1-x(n)) .
$$

Thus $s_{n+1}^{x}$ is the first $n+1$ bits of $x$ while $t_{n+1}^{x}$ is the first $n$ bits of $x$ followed by the opposite bit $1-x(n)$. Define $y_{x} \in \mathbb{R}^{\omega}$ by

$$
y_{x}(m)=\left\{\begin{aligned}
\epsilon_{n+1} & \text { if } m \in F_{s_{n+1}^{x}} \text { for some } n \\
-\epsilon_{n+1} & \text { if } m \in F_{t_{n+1}^{x}} \text { for some } n \\
\sqrt{2} \cdot \epsilon_{0} & \text { if } m \in F_{\langle\rangle} \\
0 & \text { otherwise }
\end{aligned}\right.
$$

First note that $y_{x} \in l_{p}$ for any $p>2$ :

$$
\sum\left|y_{x}(m)\right|^{p}=\left(\sqrt{2} \epsilon_{0}\right)^{p} \cdot k_{0}+\sum\left(\epsilon_{n+1}^{p}\left\|F_{s_{n+1}}\right\|+\epsilon_{n+1}^{p}\left\|F_{t_{n+1}}\right\|\right)
$$

and

$$
\sum\left(\epsilon_{n+1}^{p}\left\|F_{s_{n+1}}\right\|+\epsilon_{n+1}^{p}\left\|F_{t_{n+1}}\right\|\right)=\sum 2 \epsilon_{n+1}^{p} k_{n+1} .
$$

But for all but finitely many $n$ we have that $p_{n}<p$ and so $\epsilon_{n}^{p} k_{n}<\frac{1}{n^{2}}$.

Now we see that for distinct $x, x^{\prime} \in 2^{\omega}$ that $y_{x}$ and $y_{x^{\prime}}$ are orthogonal. Take $N<\omega$ so that $\left.x\right|_{N}=\left.x^{\prime}\right|_{N}$ but $x(N) \neq x^{\prime}(N)$. Thus $s_{n}^{x}=s_{n}^{x^{\prime}}$ and $t_{n}^{x}=t_{n}^{x^{\prime}}$ for $n \leq N$, but $s_{N+1}^{x}=t_{N+1}^{x^{\prime}}$ and $t_{N+1}^{x}=s_{N+1}^{x^{\prime}}$. Therefore

$$
\left(y_{x}, y_{x^{\prime}}\right)=\left(\sum_{n \leq N} 2 \epsilon_{n}^{2} k_{n}\right)-2 \epsilon_{N+1}^{2} k_{N+1}=\left(\sum_{n \leq N} 2 r_{n}\right)-2 r_{N+1}=0 .
$$

\section{Proof of Theorem 2.}


Kunen built a perfect set of pairwise orthogonal elements of $\mathbb{R}^{\omega}$ using a different method. His example is illustrated in figure 2. The next eight levels of his tree use the weights $\sqrt{8}$ and $-\sqrt{8}$ and so on.

Let $T \subseteq \mathbb{R}^{<\omega}$ be Kunen's tree. First note that for any $n \in \omega$,

$$
T_{n}=\{s \in T:\|s\|=n\}
$$

consists of $n$ pairwise orthogonal elements of $\mathbb{R}^{n}$. (This fact was pointed out by Abian.) Hence each $T_{n}$ is a maximal orthogonal family in $\mathbb{R}^{n}$. Now suppose $x \in \mathbb{R}^{\omega}$ is any nontrivial element. Then there exists $n \in \omega$ such that $x \uparrow n$ is nontrivial. Choose any $s \in T_{n}$ such that $(s, x \uparrow n) \neq 0$. Suppose for example that $(s, x \uparrow n)>0$ (if its negative a similar argument works). Note that for any $t \in T$ either

- $t^{\wedge} 0 \in T$ or

- there exists $w>0$ such that $t^{\wedge} w \in T$ and $t^{\wedge}-w \in T$.

In other words if a node doesn't split it continues with 0 and if it does split, then one way is positive and other negative. Using this it is easy to construct a sequence

$$
s=s_{0} \subseteq s_{1} \subseteq \cdots \subseteq s_{m} \subseteq \cdots
$$

with $s_{m} \in T_{n+m}$ such that for $m>0$

$$
s_{m}(n+m-1) \cdot x(n+m-1) \geq 0 .
$$

It follows that if $b=\cup\left\{s_{m}: m \in \omega\right\}$ and $(x, b)$ converges, then

$$
(x, b) \geq(x \uparrow n, s)>0,
$$

and so, in any case, $x$ is not orthogonal to $b$.

\section{Proof of Theorem 3 .}

For $x \in \mathbb{R}^{\omega}$ define the support of $x$ :

$$
\operatorname{supp}(x)=\{n \in \omega: x(n) \neq 0\} .
$$

Let us say that $x$ and $y$ are strongly orthogonal iff $\operatorname{supp}(x)$ and $\operatorname{supp}(y)$ are almost disjoint (i.e., intersection is finite) and $(x, y)=0$. Let $S$ be all the elements of $\mathbb{R}^{\omega}$ with infinite support. Let $S^{*}$ be all elements $x$ of $S$ such that if $n \in \operatorname{supp}(x)$ then $|x(n)|>1$. Let $\mathbb{P}$ be any countable partially ordered set. 


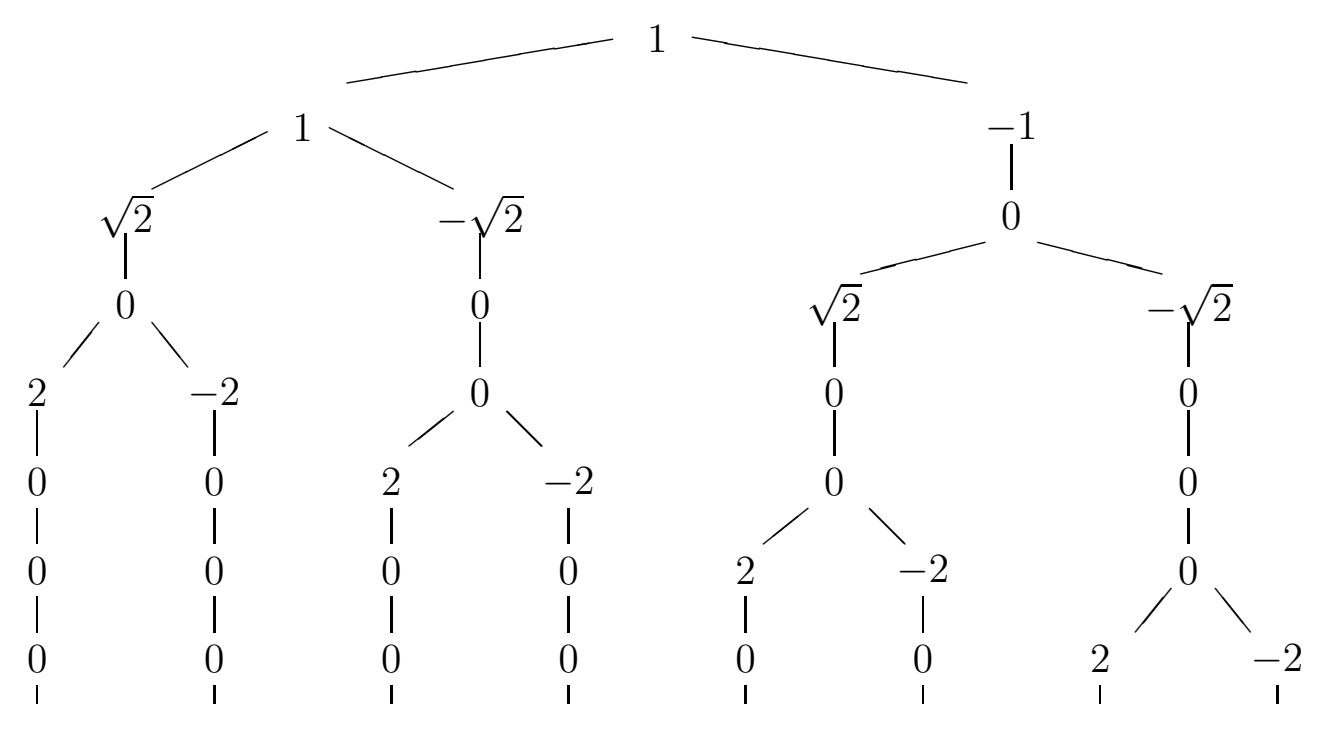

Figure 2: Kunen's perfect tree

Lemma 8 Suppose $\left\{x_{n}: n \in \omega\right\} \subseteq S^{*}$ are pairwise strongly orthogonal. Let $p \in \mathbb{P}$ and $\tau$ be a $\mathbb{P}$-name such that

$$
p \Vdash \tau \in S \text { and } \forall n\left(x_{n}, \tau\right)=0 \text {. }
$$

Then there exists $q \leq p$ and $y \in S^{*}$ such that

$$
q \Vdash(y, \tau) \neq 0
$$

and $y$ is strongly orthogonal to $x_{n}$ for every $n \in \omega$.

\section{Proof:}

Case 1. $p \Vdash \vdash^{\prime} \operatorname{supp}(\tau) \backslash \cup_{n<N} \operatorname{supp}\left(x_{n}\right)$ is infinite for each $N<\omega "$.

In this case we will take $q=p$. Let $\left\{p_{n}: n \in \omega\right\}=\{q \in \mathbb{P}: q \leq p\}$ where each element is listed with infinitely many repetitions. Build sequences

$$
k_{n} \in \omega \backslash \cup_{m<n} \operatorname{supp}\left(x_{m}\right)
$$

and

$$
l_{n}, r_{n} \in \operatorname{supp}\left(x_{n}\right) \backslash \cup_{m<n} \operatorname{supp}\left(x_{m}\right)
$$


such that the sets $\left\{k_{n}: n \in \omega\right\},\left\{l_{n}: n \in \omega\right\}$, and $\left\{r_{n}: n \in \omega\right\}$ are disjoint and such that for every $n$ there exists $q \leq p_{n}$ and $u_{n}>1$ such that

$$
q \Vdash\left|u_{n} \cdot \tau\left(k_{n}\right)\right|>1 \text {. }
$$

Now construct $y \in S^{*}$ so that

$$
\operatorname{supp}(y)=\left\{k_{n}: n \in \omega\right\} \cup\left\{l_{n}: n \in \omega\right\} \cup\left\{r_{n}: n \in \omega\right\}
$$

and $y\left(k_{n}\right)=u_{n}$ and $\left(y, x_{n}\right)=0$ for all $n$. $\left[\left(y, x_{n}\right)=0\right.$ is accomplished by picking the values of $y\left(l_{n}\right)$ and $y\left(r_{n}\right)$ inductively. Use that given $x, u, v \in \mathbb{R}$ with $u \neq 0$ and $v \neq 0$ we can pick $a, b \in \mathbb{R}$ with $|a|>1$ and $|b|>1$ and $a u+b v=x$. This is possible because if we let $b=\frac{x-a u}{v}$, then as $a \rightarrow \infty$ we have $|b| \rightarrow \infty$.]

But note that

$$
p \mid \vdash\left\|\left\{n:\left|\tau\left(k_{n}\right) y\left(k_{n}\right)\right|>1\right\}\right\|=\omega
$$

hence we are done with case 1.

Case 2. There exists $r \leq p, N<\omega$, and $F$ finite such that

$$
r \Vdash \operatorname{supp}(\tau) \subseteq \cup_{n<N} \operatorname{supp}\left(x_{n}\right) \cup F .
$$

In this case find $s \leq r$ and $n_{0}<N$ such that

$$
s\left\||| \operatorname{supp}(\tau) \cap \operatorname{supp}\left(x_{n_{0}}\right)\right\|=\omega
$$

Let $G$ be a $\mathbb{P}$-filter containing $s$. Let $Q$ be the infinite set

$$
Q=\left(\operatorname{supp}\left(x_{n_{0}}\right) \cap \operatorname{supp}\left(\tau^{G}\right)\right) \backslash \cup_{n<N, n \neq n_{0}} \operatorname{supp}\left(x_{n}\right) .
$$

Since $\tau^{G}$ is orthogonal to $x_{n_{0}}$ and $\left|x_{n_{0}}(n)\right|>1$ for each $n \in \operatorname{supp}\left(x_{n_{0}}\right)$, we must be able to find distinct $k_{1}, k_{2} \in Q$ such that the vectors $\left\langle\tau^{G}\left(k_{1}\right), \tau^{G}\left(k_{2}\right)\right\rangle$ and $\left\langle x_{n_{0}}\left(k_{1}\right), x_{n_{0}}\left(k_{2}\right)\right\rangle$ are not parallel. [Else $\left\langle\tau^{G}(k): k \in Q\right\rangle$ would be parallel to $\left\langle x_{n_{0}}(k): k \in Q\right\rangle$ and this would give that $\left(x_{n_{0}}, \tau\right) \neq 0$.] Thus we may find a pair of real numbers $u, v\left(\right.$ take $\left.\langle u, v\rangle=\left\langle x_{n_{0}}\left(k_{2}\right),-x_{n_{0}}\left(k_{1}\right)\right\rangle\right)$ such that $|u|>1$ and $|v|>1$ with $u x_{n_{0}}\left(k_{1}\right)+v x_{n_{0}}\left(k_{2}\right)=0$ but $u \tau^{G}\left(k_{1}\right)+v \tau^{G}\left(k_{2}\right) \neq 0$. Now build (similarly to case 1) $y \in S^{*}$ strongly orthogonal to each $x_{n}$ and such that $y\left(k_{1}\right)=u, y\left(k_{2}\right)=v$ and

$$
\left\{k_{1}, k_{2}\right\}=\operatorname{supp}(y) \cap\left(\cup_{n<N} \operatorname{supp}\left(x_{n}\right) \cup F\right) .
$$


Let $q \leq s$ be in $G$ such that

$$
q \Vdash u \tau\left(k_{1}\right)+v \tau\left(k_{2}\right) \neq 0 .
$$

Hence $q \Vdash(y, \tau) \neq 0$, so the lemma is proved.

Finally we prove Theorem 3 .

In Miller [3] it is shown that in the Cohen real model the following compactness-like principle holds:

For any Polish space $X$ and family of Borel sets $\left\{B_{\alpha}: \alpha<\kappa\right\}$ where $\omega_{1}<\kappa<\mathfrak{c}$, if for every $Q \subseteq \kappa$ with $\|Q\|<\kappa$ we have $\cap\left\{B_{\alpha}: \alpha \in Q\right\} \neq \emptyset$, then $\cap\left\{B_{\alpha}: \alpha<\kappa\right\} \neq \emptyset$.

It is just stated in [3] for the real line $\mathbb{R}$ but obviously it holds for any Borel image of $\mathbb{R}$. Note that for any $x \in \mathbb{R}^{\omega}$, the set

$$
B_{x}=\left\{y \in \mathbb{R}^{\omega} \backslash\{\mathbf{0}\}: x \text { is orthogonal to } y\right\}
$$

is Borel. Suppose $\left\{x_{\alpha}: \alpha<\kappa\right\}$ is a pairwise orthogonal set with $\omega_{1}<\kappa<\mathfrak{c}$. Then for any $Q \subseteq \kappa$ of with $|Q|<\kappa$ we have

$$
x_{\beta} \in \cap\left\{B_{x_{\alpha}}: \alpha \in Q\right\}
$$

for any $\beta \in \kappa \backslash Q$. By the compactness-like principle

$$
\cap\left\{B_{x_{\alpha}}: \alpha<\kappa\right\} \neq \emptyset
$$

and hence $\left\{x_{\alpha}: \alpha<\kappa\right\}$ is not maximal.

The argument for getting an maximal orthogonal set of size $\omega_{1}$ is similar to the proof that in the Cohen real model there is a maximal almost disjoint family of size $\omega_{1}$. See Kunen [2].

The problem is to construct a maximal orthogonal set in the ground model of $\mathrm{CH}$ which is not destroyed by adding one Cohen real. To begin with take $\left\{x_{n}: n \in \omega\right\}$ in $S^{*}$ which are pairwise strongly orthogonal and such that for every $m<n$ there exists a $k$ with $x_{k}(m)=1$ and $x_{k}(i)=0$ for every $i<n$ with $i \neq m$. These guarantee that no finite support element of $\mathbb{R}^{\omega}$ is orthogonal to every $x_{\alpha}$. Using the continuum hypothesis in the ground model list pairs $\left(p_{\alpha}, \tau_{\alpha}\right)$ for $\omega \leq \alpha<\omega_{1}$ of elements of $\mathbb{P} \times N$ where 
$N$ are nice $\mathbb{P}$-names for potential elements $\mathbb{R}^{\omega}$. Build $x_{\alpha}$ for $\alpha<\omega_{1}$ which are pairwise strongly orthogonal elements of $S^{*}$ such that for every $\alpha \geq \omega$ if $\left(p_{\alpha}, \tau_{\alpha}\right)$ satisfies that

$$
p_{\alpha} \Vdash \tau_{\alpha} \in S \text { and } \forall \beta<\alpha\left(x_{\beta}, \tau_{\alpha}\right)=0,
$$

then using the Lemma, there exists $q \leq p_{\alpha}$ such that

$$
q \Vdash\left(x_{\alpha}, \tau_{\alpha}\right) \neq 0 .
$$

It follows from this that $\left\{x_{\alpha}: \alpha<\omega_{1}\right\}$ remains a maximal orthogonal set when any number of Cohen reals are added. This proves Theorem 3 .

\section{Proof of Theorem 4 .}

The proof uses a modification of a partial order due to Hechler [1] used to prove the same result for MAD families.

Let $\mathbb{Q}$ denote the rational numbers. For $\gamma$ an infinite ordinal define $\mathbb{P}_{\gamma}$ as follows. An element of $\mathbb{P}_{\gamma}$ has the form $p=\left(\left\langle s_{\alpha}: N \rightarrow \mathbb{Q}, P_{\alpha}\right\rangle: \alpha \in F\right)$ where

- $N<\omega$ and $F \in[\gamma]^{<\omega}$,

- $s_{\alpha}(n) \neq 0$ implies $\left|s_{\alpha}(n)\right| \geq 1$,

- (orthogonality) $\alpha \neq \beta \in F$ implies

$$
\sum_{n<N} s_{\alpha}(n) s_{\beta}(n)=0
$$

- $P_{\alpha} \subseteq(F \cap \alpha) \times N$,

- (almost disjoint support) $(\beta, n) \in P_{\alpha}$ and $n \leq m<N$ implies

$$
s_{\alpha}(m)=0 \text { or } s_{\beta}(m)=0 .
$$

We define $p \leq q$ iff $F_{p} \supseteq F_{q}, N^{p} \geq N^{q}$, and $s_{\alpha}^{p} \uparrow N^{q}=s_{\alpha}^{q}$ and $P_{\alpha}^{p} \supseteq P_{\alpha}^{q}$ for every $\alpha \in F_{q}$. If $G$ is a sufficiently generic $\mathbb{P}_{\gamma}$-filter, then define $x_{\alpha}: \omega \rightarrow \mathbb{Q}$ by

$$
x_{\alpha}(n)=r \text { iff } \exists p \in G\left(n<N^{p} \text { and } s_{\alpha}^{p}(n)=r\right) .
$$

Note that $(\beta, n) \in P_{\alpha}$ is a promise that $\operatorname{supp}\left(x_{\alpha}\right) \cap \operatorname{supp}\left(x_{\beta}\right) \subseteq n$. Thus the $x_{\alpha}$ will be pairwise orthogonal elements of $\mathbb{Q}^{\omega}$ with almost disjoint support. 
Lemma 9 Suppose $p=\left(\left\langle s_{\alpha}: N \rightarrow \mathbb{Q}, P_{\alpha}\right\rangle: \alpha \in F\right)$ is a precondition, i.e., it satisfies everything except the orthogonality condition, but satisfies instead:

(weak orthogonality) $\alpha \neq \beta \in F$ implies either

$$
\sum_{n<N} s_{\alpha}(n) s_{\beta}(n)=0,
$$

or

$$
\alpha \notin \operatorname{dom}\left(P_{\beta}\right) \text { and } \beta \notin \operatorname{dom}\left(P_{\alpha}\right) .
$$

Then $p$ can be extended to a condition in $\mathbb{P}_{\gamma}$.

\section{Proof:}

First list all pairs $\left\{\left\langle\alpha_{n}, \beta_{n}\right\rangle: n<l\right\} \subseteq[F]^{2}$ such that

$$
\sum_{i<N} s_{\alpha_{n}}(i) s_{\beta_{n}}(i) \neq 0 \text {. }
$$

Then construct $s_{\alpha}^{n}: N_{n} \rightarrow \mathbb{Q}$ with

- $s_{\alpha}^{p}=s_{\alpha}^{0}$ for $\alpha \in F$,

- $N_{n+1}=N_{n}+2$, and

- $s_{\alpha}^{n+1} \uparrow N_{n}=s_{\alpha}^{n}$ for $\alpha \in F$,

as follows. Let

$$
x=\sum_{i<N_{n}} s_{\alpha_{n}}^{n}(i) s_{\beta_{n}}^{n}(i) .
$$

Choose $u, v \in \mathbb{Q}$ with $|u|,|v| \geq 1$ and $u+v=-x$. Now define

$$
\begin{gathered}
s_{\alpha_{n}}^{n+1}\left(N_{n}\right)=s_{\alpha_{n}}^{n+1}\left(N_{n+1}\right)=1, \\
s_{\beta_{n}}^{n+1}\left(N_{n}\right)=u \text { and } s_{\beta_{n}}^{n+1}\left(N_{n+1}\right)=v,
\end{gathered}
$$

and for all other $\delta \in F$ define

$$
s_{\delta}^{n+1}\left(N_{n}\right)=s_{\delta}^{n+1}\left(N_{n+1}\right)=0 .
$$

Now it is easy to check that

$$
\left(\left\langle s_{\alpha}^{l}: N_{l} \rightarrow \mathbb{Q}, P_{\alpha}\right\rangle: \alpha \in F\right)
$$

is a condition in $\mathbb{P}_{\gamma}$. 
Lemma $10 \mathbb{P}_{\gamma}$ has ccc, in fact, property $K$.

\section{Proof:}

Property $\mathrm{K}$ means that every uncountable set of conditions contains an uncountable subset of pairwise compatible conditions.

Given $\Gamma$ an uncountable subset of $\mathbb{P}_{\gamma}$ apply a $\Delta$-system argument to find $\Sigma \in[\Gamma]^{\omega_{1}}$ and $F$ such that $F=F^{p} \cap F^{q}=F$ for all distinct $p$ and $q$ in $\Sigma$. Next cutting down $\Sigma$ we may assume that there exists $N$ such that $N^{p}=N$ for all $p \in \Sigma$ and there are $\left(s_{\alpha}: \alpha \in F\right)$ such that

$$
\left(s_{\alpha}^{p}: \alpha \in F\right)=\left(s_{\alpha}: \alpha \in F\right)
$$

for all $p \in \Sigma$. Now for any $p, q \in \Sigma$ define $r$ by:

$$
F^{r}=F^{p} \cup F^{q}
$$

and

$$
\left\langle s_{\alpha}^{r}, P_{\alpha}^{r}\right\rangle= \begin{cases}\left\langle s_{\alpha}, P_{\alpha}^{p} \cup P_{\alpha}^{q}\right\rangle & \text { if } \alpha \in F \\ \left\langle s_{\alpha}^{p}, P_{\alpha}^{p}\right\rangle & \text { if } \alpha \in F^{p} \backslash F \\ \left\langle s_{\alpha}^{q}, P_{\alpha}^{q}\right\rangle & \text { if } \alpha \in F^{q} \backslash F\end{cases}
$$

The almost disjoint support condition holds for $r$ since it held for $p$ and $q$. For all pairs $\alpha, \beta \in F^{r} s_{\alpha}^{r}$ and $s_{\beta}^{r}$ are orthogonal except possibly those pairs with $\alpha \in F^{p} \backslash F$ and $\beta \in F^{q} \backslash F$. But these pairs satisfy the weak orthogonality condition and so by Lemma 9, $r$ can be extended to a condition $\hat{r}$ and clearly $\hat{r} \leq p$ and $\hat{r} \leq q$, and so $p$ and $q$ are compatible.

Lemma 11 Suppose $G$ is $\mathbb{P}_{\gamma}$-generic over $M$ and $\beta<\gamma$, then $G \cap \mathbb{P}_{\beta}$ is $\mathbb{P}_{\beta}$-generic over $M$.

\section{Proof:}

Actually $\mathbb{P}_{\beta}$ is what some author's call a completely embedded suborder of $\mathbb{P}_{\gamma}$. This means that for every $A \subseteq \mathbb{P}_{\beta}$ if $A$ is a maximal antichain of $\mathbb{P}_{\beta}$, then $A$ is a maximal antichain of $\mathbb{P}_{\gamma}$.

First note that if $p, q \in \mathbb{P}_{\beta}$ are incompatible in $\mathbb{P}_{\beta}$, then they are incompatible in $\mathbb{P}_{\gamma}$. This is because if $r \leq p$ and $r \leq q$ then define $r \mid \beta \in \mathbb{P}_{\beta}$ by

$$
r \vdash \beta=\left(\left\langle s_{\alpha}^{r}, P_{\alpha}^{r}\right\rangle: \alpha \in F^{r} \cap \beta\right) .
$$


Then $r \uparrow \beta \leq p$ and $r \uparrow \beta \leq q$.

Claim. If $p \in \mathbb{P}_{\beta}$ and $r \in \mathbb{P}_{\gamma}$ are incompatible in $\mathbb{P}_{\gamma}$, then $p$ and $r \uparrow \beta$ are incompatible.

Else suppose there exists $q \in \mathbb{P}_{\beta}$ with $q \leq p$ and $q \leq r \uparrow \beta$ and without loss assume $N^{q}>N^{r}$. For $\alpha \in F^{r} \backslash \beta$ define $\hat{s}_{\alpha}: N^{q} \rightarrow \mathbb{Q}$ by $\hat{s}_{\alpha} \uparrow N^{r}=s_{\alpha}^{r}$ and $\hat{s}_{\alpha}(n)=0$ for all $n$ with $N^{r} \leq n<N^{q}$. Consider the precondition $t$ defined by

$$
F^{t}=F^{r} \cup F^{q}
$$

and

$$
\left\langle s_{\alpha}^{t}, P_{\alpha}^{t}\right\rangle= \begin{cases}\left\langle\hat{s}_{\alpha}, P_{\alpha}^{r}\right\rangle & \text { if } \alpha \in F^{r} \backslash \beta \\ \left\langle s_{\alpha}^{q}, P_{\alpha}^{q}\right\rangle & \text { if } \alpha \in F^{q}\end{cases}
$$

But then $t$ extends to a condition by Lemma 9, showing that $p$ and $r$ are compatible and proving the Claim.

It follows from the Claim that if $A \subseteq \mathbb{P}_{\beta}$ is a maximal antichain of $\mathbb{P}_{\beta}$, then $A$ is a maximal antichain of $\mathbb{P}_{\gamma}$, and hence the lemma is proved.

Lemma 12 Let $\gamma$ be a limit ordinal and suppose $\tau$ is a $\mathbb{P}_{\gamma}$-name for an element of $\mathbb{R}^{\omega}, F \in[\gamma]^{<\omega}, H \in \omega$, and $p \in \mathbb{P}_{\gamma}$ have the property that

$$
p \mid \vdash\|\operatorname{supp}(\tau)\|=\omega \text { and } \operatorname{supp}(\tau) \subseteq \bigcup_{\beta \in F} \operatorname{supp}\left(x_{\beta}\right) \cup H .
$$

Then there exists $\alpha_{1} \in \gamma$ and $q \leq p$ such that

$$
q \Vdash\left(\tau, x_{\alpha_{1}}\right) \neq 0 \text {. }
$$

\section{Proof:}

Without loss we may assume for every $\alpha \in F$

$$
p \mid \vdash\left(\tau, x_{\alpha}\right)=0 .
$$

Find $r \leq p$ and $\alpha_{0} \in F$ such that

$$
r \Vdash \operatorname{supp}(\tau) \cap \operatorname{supp}\left(x_{\alpha_{0}}\right) \text { infinite. }
$$

Let $G$ be $\mathbb{P}_{\gamma}$-generic with $r \in G$ and let $Q$ be the infinite set defined by

$$
Q=\left(\operatorname{supp}\left(\tau^{G}\right) \cap \operatorname{supp}\left(x_{\alpha_{0}}\right)\right) \backslash\left(H \cup \bigcup\left\{\operatorname{supp}\left(x_{\beta}\right): \beta \in F, \beta \neq \alpha_{0}\right\}\right) .
$$


Then there must be $k_{0}<k_{1} \in Q$ such that $\left\langle\tau^{G}\left(k_{0}\right), \tau^{G}\left(k_{1}\right)\right\rangle$ is not parallel to $\left\langle x_{\alpha_{0}}\left(k_{0}\right), x_{\alpha_{0}}\left(k_{1}\right)\right\rangle$. Take $t \leq r$ with $t \in G$ with $H \leq k_{0}<k_{1}<N^{t}-1$ and $s_{\alpha}^{t}\left(k_{0}\right)=u$ and $s_{\alpha}^{t}\left(k_{1}\right)=v$ and such that

$$
t \Vdash v \cdot \tau\left(k_{0}\right)+(-u) \cdot \tau\left(k_{1}\right) \neq 0 .
$$

Note that since $t \in G$, for any $\beta \in F \backslash\left\{\alpha_{0}\right\}$ we have that $s_{\beta}^{t}\left(k_{i}\right)=0$. Choose any $\alpha_{1}>\max \left(F^{t}\right)$ and define the precondition $q$ as follows:

$$
\begin{gathered}
F^{q}=F^{t} \cup\left\{\alpha_{1}\right\}, \\
\left(\left\langle s_{\alpha}^{q}, P_{\alpha}^{q}\right\rangle: \alpha \in F^{t}\right)=\left(\left\langle s_{\alpha}^{t}, P_{\alpha}^{t}\right\rangle: \alpha \in F^{t}\right),
\end{gathered}
$$

and define $s_{\alpha_{1}}: N^{t} \rightarrow \mathbb{Q}$ by

$$
s_{\alpha_{1}}(l)=\left\{\begin{aligned}
v & \text { if } l=k_{0} \\
-u & \text { if } l=k_{1} \\
0 & \text { otherwise }
\end{aligned}\right.
$$

Define

$$
P_{\alpha_{1}}^{q}=\left\{(0, \beta): \beta \in F \backslash\left\{\alpha_{0}\right\}\right\} \cup\left\{\left(N^{t}-1, \alpha_{0}\right)\right\} .
$$

The precondition $q$ satisfies all requirements to be an element of $\mathbb{P}_{\gamma}$ except possibly the orthogonality condition. But note that for $\beta \in F$

$$
\sum_{n<N^{t}} s_{\alpha_{1}}(n) s_{\beta}(n)=0 .
$$

So we only need to worry about $\beta \in F^{t} \backslash F$ and $\alpha_{1}$. But for these

$$
\alpha_{1} \notin \operatorname{dom}\left(P_{\beta}^{q}\right) \text { and } \beta \notin \operatorname{dom}\left(P_{\alpha_{1}}^{q}\right)
$$

and so we can extend $q$ to a condition using Lemma 9. Let us denote this extension also by $q$. By the definition of $P_{\alpha_{1}}^{q}$ we have that

$$
q \Vdash \operatorname{supp}(\tau) \cap \operatorname{supp}\left(x_{\alpha_{1}}\right)=\left\{k_{0}, k_{1}\right\}
$$

and thus

$$
q \Vdash\left(\tau, x_{\alpha_{1}}\right)=v \cdot \tau\left(k_{0}\right)+(-u) \cdot \tau\left(k_{1}\right) \neq 0 .
$$


Lemma 13 Suppose $\tau$ is a $\mathbb{P}_{\gamma}$-name for an element of $\mathbb{R}^{\omega}$, and let $p \in \mathbb{P}_{\gamma+1}$ have the property that for every $F \in[\gamma]^{<\omega}$

$$
p \Vdash\left\|\operatorname{supp}(\tau) \backslash \bigcup_{\beta \in F} \operatorname{supp}\left(x_{\beta}\right)\right\|=\omega .
$$

Then

$$
p|\vdash| \tau(n) \cdot x_{\gamma}(n) \mid \geq 1 \text { for infinitely many } n \text {. }
$$

\section{Proof:}

Suppose not and let $q \leq p$ and $N<\omega$ be such that

$$
q \Vdash \forall n>N\left|\tau(n) \cdot x_{\gamma}(n)\right|<1 .
$$

Let $G$ be $\mathbb{P}_{\gamma+1}$-generic over $M$ with $q \in G$. Choose $n>N, N^{q}$ with

$$
n \in \operatorname{supp}\left(\tau^{G}\right) \backslash \cup\left\{\operatorname{supp}\left(x_{\alpha}\right): \alpha \in F^{q} \cap \gamma\right\} .
$$

Since $\tau$ is a $\mathbb{P}_{\gamma}$-name and $G \cap \mathbb{P}_{\gamma}$ is $\mathbb{P}_{\gamma^{-}}$-generic over $M$ there exists $r \in G \cap \mathbb{P}_{\gamma}$ and $m \in \omega$ such that

$$
r|\vdash| \tau(n) \mid>\frac{1}{m+1} .
$$

We may assume without loss of generality that $N^{r}>n$ and $r \leq q \uparrow \gamma$. Note that $s_{\alpha}^{r}(n)=0$ for all $\alpha \in F^{q} \cap \gamma$. Now define $\hat{s}_{\gamma}: N^{r} \rightarrow \mathbb{Q}$ as follows.

$$
\hat{s}_{\gamma}(k)= \begin{cases}s_{\gamma}^{q}(k) & \text { if } k<N^{q} \\ m+1 & \text { if } k=n \\ 0 & \text { otherwise }\end{cases}
$$

Let

$$
t=\left(\left\langle s_{\alpha}^{r}, P_{\alpha}^{r}\right\rangle: \alpha \in F^{r}\right) \cup\left(\hat{s}_{\gamma}: P_{\gamma}^{q}\right) .
$$

The precondition $t$ satisfies the weak orthogonality condition of Lemma 9 and thus can be extended to a condition in $\mathbb{P}_{\gamma}$. But it would then force $\left|x_{\gamma}(n) \cdot \tau(n)\right|>1$ which would be a contradiction.

Lemma 14 Suppose $G$ is $\mathbb{P}_{\gamma}$-generic over $M(\operatorname{cof}(\gamma)>\omega)$ and $\left\{x_{\alpha}: \alpha<\gamma\right\}$ are the generic family of mutually orthogonal elements of $\mathbb{Q}^{\omega}$, then in $M[G]$ for every $y \in \mathbb{R}^{\omega}$ there exists $\alpha<\gamma$ such that $y$ and $x_{\alpha}$ are orthogonal. 


\section{Proof:}

First note that by any easy density argument for any $m<n<\omega$ there are infinitely many $k<\omega$ such that

$$
x_{k}(j)= \begin{cases}1 & \text { if } j=m \\ 0 & \text { if } j<n \text { and } j \neq m .\end{cases}
$$

Hence we need not worry about $y$ with finite support. If there exists

$$
F \in[\gamma]^{<\omega} \text { and } H<\omega
$$

such that

$$
\operatorname{supp}(y) \subseteq\left(H \cup \bigcup\left\{\operatorname{supp}\left(x_{\alpha}\right): \alpha \in F\right\}\right),
$$

then $y$ is taken care of by Lemma 12. On the other hand if there is no such $F$, then by using ccc and the fact that $\operatorname{cof}(\gamma)$ is uncountable, we can find $\delta<\gamma$ and a $\mathbb{P}_{\delta}$-name $\tau$ for $y$ such that the hypothesis of Lemma 13 holds and thus $\left(x_{\delta}, y\right) \neq 0$.

Finally we prove Theorem 4 . Force with the finite support product

$$
\sum\left\{\mathbb{P}_{\gamma}: \gamma<\kappa\right\}
$$

This product has property $\mathrm{K}$ since each of its factors does. Since this partial order has cardinality $\kappa$ and $\kappa^{\omega}=\kappa$ in $M$, the continuum has cardinality $\kappa$ in the generic extension. Also by the product lemma, if $\left\langle G_{\gamma}: \gamma<\kappa\right\rangle$ is $\sum\left\{\mathbb{P}_{\gamma}: \gamma<\kappa\right\}$-generic over $M$, then for each $\gamma_{0}<\kappa$ we have that $G_{\gamma_{0}}$ is $\mathbb{P}_{\gamma_{0}}$-generic over

$$
M\left[G_{\gamma}: \gamma<\kappa, \gamma \neq \gamma_{0}\right]
$$

Hence for each ordinal $\gamma<\kappa$ of uncountable cofinality we have a maximal

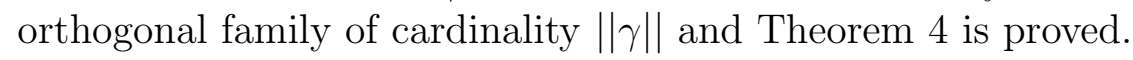

\section{Proof of Theorem 5.}

For simplicity we first present a proof for the case when $X$ is disjoint from $l_{2}$. Let $\mathbb{P}$ be the following poset. An element of $\mathbb{P}$ has the form

$$
p=(s: N \rightarrow \mathbb{Q}, F, P)
$$


where $N<\omega, F \in[X]^{<\omega}$, and $P$ is a finite set of requirements of the form $P \subseteq F \times(N+1) \times \mathbb{Q}^{+}$where $\mathbb{Q}^{+}$is the positive rationals and for every $(x, k, \epsilon) \in P$,

$$
\left|\sum_{n<k} s(n) \cdot x(n)\right|<\epsilon
$$

and for every $l$ with $k<l \leq N$

$$
\left|\sum_{k \leq n<l} s(n) \cdot x(n)\right|<\epsilon .
$$

We define $p \leq q$ iff $N^{q} \leq N^{p}, q=p \uparrow N^{q}, F^{q} \subseteq F^{p}$, and $P^{q} \subseteq P^{p}$. The poset $\mathbb{P}$ is $\sigma$-centered, because two conditions with the same $s$ are compatible.

Lemma 15 For any $p \in \mathbb{P}, x \in F$, and $\epsilon \in \mathbb{Q}^{+}$there exists $q \leq p$, and $k<\omega$ such that $(x, k, \epsilon) \in P^{q}$.

\section{Proof:}

Let $p=(s: N \rightarrow \mathbb{Q}, F, P)$. Let $\epsilon_{0}>0$ be such that for any $(y, k, \gamma) \in P$

$$
\left|\sum_{k \leq n<N} s(n) \cdot y(n)\right|+\epsilon_{0}<\gamma
$$

Choose $N_{0} \geq N$ so that for any $m>N_{0}$ and $y \in F \backslash\{x\}$

$$
\left|\sum_{N_{0} \leq n<m} x(n) \cdot y(n)\right|<\epsilon_{0} .
$$

Let

$$
b=\sum_{n<N} x(n) \cdot s(n) .
$$

Let $N_{1}>N_{0}$ be minimal such that

$$
\sum_{N_{0} \leq n<N_{1}} x(n)^{2}>b
$$

(This exists since $x$ is not in $l_{2}$.) Choose $\rho$ with $|\rho| \leq 1$ and so that

$$
\rho \cdot \sum_{N_{0}<n<N_{1}} x(n)^{2}=-b .
$$


Now consider $t: N_{1} \rightarrow \mathbb{R}$ defined as below:

$$
t(n)=\left\{\begin{array}{cl}
s(n) & \text { if } n<N \\
0 & \text { if } N \leq n<N_{0} \\
\rho \cdot x(n) & \text { if } N_{0} \leq n<N_{1}
\end{array}\right.
$$

For any $(y, k, \gamma) \in P$ with $y \neq x$ and $m \leq N_{1}$ note that

$$
\begin{aligned}
\left|\sum_{k \leq n<m} t(n) y(n)\right| & \leq\left|\sum_{k \leq n<N} s(n) y(n)\right|+|\rho| \cdot\left|\sum_{N_{0} \leq n<m} x(n) y(n)\right| \\
& \leq\left|\sum_{k \leq n<N} s(n) y(n)\right|+\epsilon_{0} \\
& <\gamma .
\end{aligned}
$$

Note that since $N_{1}$ was chosen minimal,

$$
\left|\sum_{k<n<m+1} t(n) \cdot x(n)\right| \leq\left|\sum_{k<n<m} t(n) \cdot x(n)\right|
$$

for any $m$ with $N_{0} \leq m<N_{1}$, consequently any requirements involving $x$ are also satisfied.

Since

$$
\sum_{n<N_{1}} t(n) \cdot x(n)=0
$$

we can change the values of $t$ on $\left[N_{0}, N_{1}\right)$ to be rational to get $t^{q}: N_{1} \rightarrow \mathbb{Q}$ so that

$$
\left|\sum_{n<N_{1}} t^{q}(n) \cdot x(n)\right|<\epsilon
$$

and still satisfy all the requirements of $P$. Letting

$$
q=\left(t^{q}, F, P \cup\left\{\left(x, N_{1}, \epsilon\right)\right\}\right)
$$

proves the lemma.

Lemma 16 For any $p \in \mathbb{P}$ and $l<\omega$, there exist $q \leq p$ such that

$$
\sum_{n<N^{q}} s^{q}(n)^{2}>l
$$




\section{Proof:}

Given $p$ just let $x \in X \backslash F^{p}$. As in the proof of Lemma 15 we can extend $p$ to equal $x$ as much as we like. Since $x$ is not in $l_{2}$ and $p$ has no requirements mentioning $x$ we can get the norm of $s^{q}$ greater than $l$.

The Lemmas show that if we define

$$
D_{l}=\left\{q \in \mathbb{P}: \sum_{n<N^{q}} s^{q}(n)^{2}>l\right\}
$$

and for each $x \in X$ and $\epsilon \in \mathbb{Q}^{+}$

$$
D_{x}^{\epsilon}=\left\{q \in \mathbb{P}: x \in F^{q} \text { and } \exists k(x, k, \epsilon) \in q\right\}
$$

then these sets are dense. Applying MA we get a $\mathbb{P}$-filter $G$ meeting them all. Then letting

$$
z=\cup\left\{s^{p}: p \in G\right\}
$$

we have that $z$ is not in $l_{2}$ but is orthogonal to every element of $X$. This proves Theorem 5 in the case that $X$ contains no elements of $l_{2}$.

Next we indicate how to modify our partial order in case $X$ contains finitely many elements of $l_{2}$. Let $H=X \cap l_{2}$ be finite. First we replace $\mathbb{Q}$ by any countable field $\mathbb{Q}^{*}$ which contains the rationals and all the coefficients of elements of $H$. We make the following two additional demands for

$$
p=\left(s: N \rightarrow \mathbb{Q}^{*}, F, P\right)
$$

to be an element of $\mathbb{P}$.

1. $H \subseteq F$, and

2. for each $x \in H$ we have $\sum_{n<N} s(n) x(n)=0$.

The side requirements $P$ are as before including the ones for elements of $H$. Now we prove Lemma 15 using our modified definition of $\mathbb{P}$. In the case that $x \in H$ this is trivial since we can simply add $(x, N, \epsilon)$ to $P^{p}$. So let us assume $x \in F \backslash H$ where $p=\left(s: N \rightarrow \mathbb{Q}^{*}, F, P\right)$. Let $\epsilon_{0}$ be such that $0<\epsilon_{0}<\epsilon$ and for any $(y, k, \gamma) \in P$

$$
\left|\sum_{k \leq n<N} s(n) \cdot y(n)\right|+\epsilon_{0}<\gamma
$$


And this time choose $N_{0} \geq N$ so that for any $m>N_{0}$ and $y \in F \backslash\{x\}$

$$
\left|\sum_{N_{0} \leq n<m} x(n) \cdot y(n)\right|<\epsilon_{0} / 2
$$

For simplicity we begin by giving the proof in the case $H$ has a single element say $H=\{z\}$. If $\operatorname{supp}(z)$ is finite, it is easy to do since we can just extend $s$ by zero until we are beyond the support of $z$ and then apply the same argument as before. So assume that the support of $z$ is infinite. Let $j>N_{0}$ be so that $z(j) \neq 0$. Choose $\delta>0$ so that

$$
\delta \cdot \max \{|y(j)|: y \in F\} \leq \epsilon_{0} / 2 .
$$

Now choose $N_{1}>j>N_{0}$ so that for every $m>N_{1}$

$$
\left|\sum_{N_{1} \leq n<m} x(n) z(n)\right|<|z(j)| \cdot \delta .
$$

As in the first proof we may find $N_{2}>N_{1}$ and $|\rho| \leq 1$ so that

$$
\sum_{n<N} x(n) s(n)+\sum_{N_{1} \leq n<N_{2}} \rho \cdot x(n) x(n)=0 .
$$

Now we define $t: N_{2} \rightarrow \mathbb{R}$ as below:

$$
t(n)=\left\{\begin{array}{cl}
s(n) & \text { if } n<N \\
0 & \text { if } N \leq n<N_{1} \text { and } n \neq j \\
\frac{-1}{z(j)} \sum_{N_{1} \leq k<N_{2}} \rho x(k) z(k) & \text { if } n=j \\
\rho \cdot x(n) & \text { if } N_{1} \leq n<N_{2}
\end{array}\right.
$$

The value of $t(j)$ is picked to make $t$ and $z \uparrow N_{2}$ orthogonal (remembering that $s$ and $z \uparrow N$ are already orthogonal). First note that $|y(j) t(j)|<\epsilon_{0} / 2$ for every $y \in F$, because $|t(j)|<\delta$ and $|y(j)| \cdot \delta \leq \epsilon_{0} / 2$. Since

$$
\left|\sum_{N_{1} \leq n<m} y(n) t(n)\right|<\epsilon_{0} / 2
$$

for all $y \in F \backslash\{x\}$ and $m \leq N_{2}$, all the requirements in $P$ are kept. Note that

$$
\left|\left(t, x \uparrow N_{2}\right)\right|=|t(j) x(j)| \leq \epsilon_{0} / 2
$$


Now we set $P^{t}=P \cup\left\{\left(x, N_{2}, \epsilon\right)\right\}$ and $F^{t}=F$. The final step is to change the values of $t$ on $j$ and $\left[N_{1}, N_{2}\right)$ to elements of $\mathbb{Q}^{*}$. First slightly perturb the values on $\left[N_{1}, N_{2}\right)$ and then use them to set the value of $t(j)$ so that

$$
t(j) z(j)=-\sum_{N_{1} \leq n<N_{2}} t(n) z(n) .
$$

This new $t$ will be orthogonal to $z \uparrow N_{2}$ and satisfy all the requirements of $P$. This concludes the proof of Lemma 15 in the case that $X \cap l_{2}$ consists of a singleton $\{z\}$.

Finally we sketch the proof of the Lemma in the case that $X \cap l_{2}=H$ is an arbitrary finite set. $N_{0}$ and $\epsilon_{0}$ are chosen as before. Begin by choosing $H_{0} \subseteq H$ so that $\left\{z \uparrow\left[N_{0}, \omega\right): z \in H_{0}\right\}$ are linearly independent (in the space $\mathbb{R}^{\left[N_{0}, \omega\right)}$ ) and so that $\left\{z \uparrow\left[N_{0}, \omega\right): z \in H\right\}$ is contained in the span of $\left\{z \uparrow\left[N_{0}, \omega\right): z \in H_{0}\right\}$. Choose $N_{1}>N_{0}$ so that $V=\left\{z \uparrow\left[N_{0}, N_{1}\right): z \in H_{0}\right\}$ are linearly independent.

Claim. For any $\epsilon_{1}>0$ there exists $\delta>0$ so that for any $\left\langle\beta_{v}: v \in V\right\rangle$ with $\left|\beta_{v}\right|<\delta$ there exists $t \in \mathbb{R}^{\left[N_{0}, N_{1}\right)}$ with $\|t\|_{2}<\epsilon_{1}$ and $(v, t)=\beta_{v}$ for every $v \in V$.

Here $\|\cdot\|_{2}$ is the usual $l_{2}$ norm and $(v, t)$ the usual inner product in the finite dimensional vector space $\mathbb{R}^{\left[N_{0}, N_{1}\right)}$.

The proof of the Claim is an elementary exercise in Linear Algebra. Let $V=\left\{v_{1}, \ldots, v_{m}\right\}$ and let $W$ be the span of $V$. Define the linear map

$$
T: W \rightarrow \mathbb{R}^{m} \text { by } T(t)=\left\langle\left(t, v_{i}\right): i=1, \ldots, m\right\rangle \text {. }
$$

It follows from the Gram-Schmidt orthogonalization process that the kernel of $T$ is trivial. Hence the range of $T$ is $\mathbb{R}^{m}$. The existence of $\delta$ now follows from the continuity of $T$. This proves the Claim.

We leave the value of $\epsilon_{1}$ to be determined latter. We find $N_{2}>N_{1}$ so that for any $m>N_{2}$ and $y \in F \backslash\{x\}$

$$
\left|\sum_{N_{0} \leq n<m} x(n) \cdot y(n)\right|<\delta .
$$

As in the argument before we find $N_{3}>N_{2}$ and define $t \uparrow\left[N_{2}, N_{3}\right)$ to be a small scaler multiple of $x \uparrow\left[N_{2}, N_{3}\right)$ in such a way as to make the inner product of $x$ and $t$ zero. We now use the Claim to extend $t$ on the interval 
$\left[N_{0}, N_{1}\right)$ to make sure that for every $y \in H_{0}$ the inner product of $t$ and $y$ is zero. If we choose $\epsilon_{1}$ small enough so that

$$
\epsilon_{1} \cdot \| x\left\lceil\left[N_{1}, N_{2}\right) \|_{2}<\epsilon_{0} / 2\right.
$$

for every $x \in F$, then this $t$ works (when jiggled to have range $\mathbb{Q}^{*}$ ).

Since $\left\|t \uparrow\left[N_{1}, N_{2}\right)\right\|_{2} \cdot\left\|x \uparrow\left[N_{1}, N_{2}\right)\right\|_{2} \leq \epsilon_{0} / 2$ for every $x \in F$ all commitments from $P$ are honored. Also $t$ is orthogonal to all elements of $H$. The reason is that $t \uparrow N_{0}=s$ is already orthogonal to every $z \in H$ and every element of $\left\{z \uparrow\left[N_{0}, \omega\right): z \in H\right\}$ is contained in the span of $\left\{z \uparrow\left[N_{0}, \omega\right): z \in H_{0}\right\}$ and $t$ is orthogonal to everything in $\left\{z \uparrow\left[N_{0}, \omega\right): z \in H_{0}\right\}$.

This concludes the proof of Theorem 5 .

\section{Proof of Theorem 6 .}

For any $h<\omega$ define the partial order $\mathbb{P}_{h}$ as follows. A condition in $\mathbb{P}_{h}$ has the following form:

$$
p=\left(\left(s_{i}: N \rightarrow \mathbb{Q}^{\neq 0}: i<h\right), R\right)
$$

where $N<\omega, \mathbb{Q}^{\neq 0}$ are the nonzero rationals, and $R \subseteq[h]^{2} \times N \times \mathbb{Q}^{+}$is a finite set of requirements satisfying the following: for any $(\{i, j\}, k, \epsilon) \in R$ :

$$
\left|\sum_{n<k} s_{i}(n) \cdot s_{j}(n)\right|<\epsilon
$$

and for any $l$ with $k<l \leq N$

$$
\left|\sum_{k \leq n<l} s_{i}(n) \cdot s_{j}(n)\right|<\epsilon .
$$

Lemma 17 For any $p \in \mathbb{P}_{h}, \epsilon \in \mathbb{Q}^{+}$, and $\{i, j\} \in[h]^{2}$ there exists $q \leq p$ and $K$ such that $(\{i, j\}, K, \epsilon) \in R^{q}$.

\section{Proof:}

Let

$$
b=\sum_{n<N^{p}} s_{i}^{p}(n) \cdot s_{j}^{p}(n) .
$$


Let $N^{q}=N^{p}+1$ Define $s_{i}^{q}\left(N^{p}\right)=b$ and $s_{i}^{q}\left(N^{p}\right)=-1$. Note that for any requirement of the form $\left((\{i, j\}, k, \delta) \in R^{p}\right.$ that

$$
\sum_{k \leq n<N^{q}} s_{i}^{q}(n) \cdot s_{j}^{q}(n)=-b+\sum_{k \leq n<N^{p}} s_{i}^{p}(n) \cdot s_{j}^{p}(n)=-\sum_{n<k} s_{i}^{p}(n) \cdot s_{j}^{p}(n)
$$

so the requirement is met. Now for $l$ different from $i$ and $j$ we $s_{l}\left(N^{p}\right)$ to be some sufficiently small positive rational which is picked so as to still satisfy all the requirements of $R^{p}$. Let

$$
q=\left(\left(s_{i}^{q}: N^{p}+1 \rightarrow \mathbb{Q}^{\neq 0}: i<h\right), R^{p} \cup\left\{\left(\{i, j\}, N^{p}+1, \epsilon\right)\right\}\right) .
$$

Lemma 18 For any $h<\omega$ there exists $N<\omega$ and $r_{i}: N \rightarrow\{-1,1\}$ for $i<h$ such that $r_{i}$ and $r_{j}$ are orthogonal for any $i \neq j$.

\section{Proof:}

Let $N=2^{h}$ and identify it with the maps from $h$ into $2=\{0,1\}$. For each $i<h$ define $r_{i}: 2^{h} \rightarrow\{-1,1\}$ by

$$
r_{i}(t)=\left\{\begin{aligned}
-1 & \text { if } t(i)=1 \\
1 & \text { if } t(i)=0
\end{aligned}\right.
$$

Note that

$r_{i}(t) \cdot r_{j}(t)=-1$ iff $(t(i)=1$ and $t(j)=0)$ or $(t(i)=0$ and $t(j)=1)$.

But

$$
\|\left\{t \in 2^{h}: t(i)=1 \text { and } t(j)=0\right\} \|=\frac{1}{4} N
$$

and

$$
\|\left\{t \in 2^{h}: t(i)=0 \text { and } t(j)=1\right\} \|=\frac{1}{4} N
$$

and so the inner product of $r_{i}$ and $r_{j}$ is 0 .

Lemma 19 Suppose $p \in \mathbb{P}_{h}$ and $l<\omega$. Then there exists $q \leq p$ and $\epsilon>0$ such that $\left(N^{q}-N^{p}\right) \epsilon^{l}>1$ and for every $n$ with $N^{p} \leq n<N^{q}$ and $i<h$ we have $\left|s_{i}^{q}(n)\right|=\epsilon$. 


\section{Proof:}

Let $\delta \in \mathbb{Q}^{+}$be such that for every $(\{i, j\}, k, \gamma) \in R^{p}$ we have

$$
\left|\sum_{k \leq n<N^{p}} s_{i}^{p}(n) \cdot s_{j}^{p}(n)\right|+\delta^{2}<\gamma
$$

Apply Lemma 18 to obtain pairwise orthogonal $r_{i}: N \rightarrow\{-1,1\}$ for $i<h$. Take $\epsilon=\frac{\delta}{N}$. Now define $\hat{s}_{i}: N^{p}+N \rightarrow \mathbb{Q}$ by

$$
\hat{s}_{i}\left(N_{k}^{p}\right)(n) \begin{cases}s_{i}^{p}(n) & \text { if } n<N^{p} \\ \epsilon \cdot r_{i}(k) & \text { if } n=N^{p}+k\end{cases}
$$

Note that for any $i \neq j$ and $k<N^{p} \leq m \leq N^{p}+N$ that

$$
\left|\sum_{k<n<m} \hat{s}_{i}(n) \cdot \hat{s}_{j}(n)\right| \leq\left|\sum_{k<n<N^{p}} s_{i}^{p}(n) \cdot s_{j}^{p}(n)\right|+\epsilon^{2} \cdot N
$$

and since $\epsilon^{2} \cdot N<\delta^{2}$ any requirements involving $\{i, j\}$ are kept. Note also that

$$
\sum_{n<N^{P}+N} \hat{s}_{i}(n) \cdot \hat{s}_{j}(n)=\sum_{n<N} s_{i}(n) \cdot s_{j}(n)
$$

and so this trick can be repeated with the same $\epsilon$ as many times as is necessary to make $\left(N^{q}-N^{p}\right) \cdot \epsilon^{l}>1$.

Remark. We could have avoided the use of Lemma 18 by taking a pair of weights $\epsilon>0$ and $\delta>0$ and the column vectors of the $h \times h$ matrix with $-\delta$ on the diagonal and $\epsilon$ off the diagonal. The columns will be orthogonal provided

$$
-2 \epsilon \delta+(h-2) \epsilon^{2}=0 \text { or } \delta=\frac{h-2}{2} \epsilon
$$

In this case we would get that $\left|s_{i}^{q}(n)\right| \geq \epsilon$ in the conclusion of Lemma 19 .

Finally, to prove Theorem [6 we construct a sequence $p_{n} \in \mathbb{P}_{h_{n}}$ where $h_{n}<h_{n+1}$. Start with any $p_{0}$ and $h_{0}$. At stage $n$ given $p_{n}$ apply Lemma 17 ( $h_{n}$ times) to obtain $p \leq p_{n}$ so that for each $\{i, j\} \in\left[h_{n}\right]^{2}$ for some $k<N^{p}$ we have that $\left(\{i, j\}, k, \frac{1}{n}\right) \in R^{p}$. Now apply Lemma 19 to obtain $q \leq p$ and $\epsilon>0$ such that $\left(N^{q}-N^{p}\right) \epsilon^{n}>1$ and for every $m$ with $N^{p} \leq m<N^{q}$ and 
$i<h_{n}$ we have $\left|s_{i}^{q}(m)\right|=\epsilon$. Finally obtain $p_{n+1}$ by "doubling" $p_{n}$, i.e., let $h_{n+1}=2 \cdot h_{n}$ define

$$
s_{2 i}^{p_{n+1}}=s_{2 i+1}^{p_{n+1}}=s_{i}^{p_{n}}
$$

and

$$
R^{p_{n+1}}=\left\{(\{2 i, 2 j\}, k, \gamma),(\{2 i+1,2 j+1\}, k, \gamma):(\{i, j\}, k, \gamma) \in R^{p_{n}}\right\} .
$$

This completes the construction.[ Now define $P$ by

$$
P=\left\{x \in \mathbb{R}^{\omega}: \forall n \exists i<h_{n} x \vdash h_{n}=s_{i}^{p_{n}}\right\} .
$$

It is easy to verify that the perfect set $P$ has the properties required. This proves Theorem 6 .

\section{Proof of Theorem 7 .}

For part (a) first consider the following example:

$$
\begin{aligned}
& x_{0}=\left(\begin{array}{llllll}
1, & -1, & 0, & 0, & 0, & \ldots
\end{array}\right) \\
& x_{1}=(1, \quad 1, \quad-2, \quad 0, \quad 0, \ldots) \\
& x_{2}=(1, \quad 1, \quad 1,-3,0, \ldots) \\
& \text { : }
\end{aligned}
$$

Then $\left\{x_{n}: n<\omega\right\}$ is an orthogonal family in $l_{2}$. We claim that the only $u$ 's which are orthogonal to all of the $x_{n}$ 's are scalar multiples of $(1,1,1, \ldots)$. If $u=\left(u_{0}, u_{1}, u_{2}, \ldots\right)$ is orthogonal to all of the $x_{n}$, then

$$
\begin{aligned}
u_{0}-u_{1} & =0 \\
u_{0}+u_{1}-2 u_{2} & =0 \\
u_{0}+u_{1}+u_{2}-3 u_{3} & =0
\end{aligned}
$$

It follows easily $u_{0}=u_{1}=u_{2}=\cdots$. This is Kunen's example and it answered K.P Hart's original question.

\footnotetext{
${ }^{3}$ It is not necessary to make $s_{2 i}$ differ from $s_{2 i+1}$ since this will automatically be taken care of when we use of Lemma 17 .
} 
To get the example of part (a) do as follows. Work in $\mathbb{R}^{\omega \times \omega}$. Define $x_{m}^{n}$ as follows:

$$
x_{m}^{n}(l, j)=\left\{\begin{array}{cl}
1 & \text { if } n=l \text { and } j \leq m \\
-(m+1) & \text { if } n=1 \text { and } j=m+1 \\
0 & \text { otherwise }
\end{array}\right.
$$

So it is the example above repeated on infinitely many disjoint copies of $\omega$. Let $X=\left\{x_{m}^{n}: n, m<\omega\right\}$. Then as in Kunen's example:

$z \in \mathbb{R}^{\omega \times \omega}$ is orthogonal to all elements of $\left\{x_{m}^{n}: m<\omega\right\}$

iff

$z \uparrow\{n\} \times \omega$ is a scalar multiple of the constant 1 sequence.

Define $y_{n}: \omega \times \omega \rightarrow\{0,1\}$ to be constantly 1 on $\{n\} \times \omega$ and to be 0 otherwise. Then it is easy to check that $X \cup\left\{y_{n}: n<\omega\right\}$ is a maximal orthogonal family. Similarly for any $n$ define $v_{n}: \omega \times \omega \rightarrow\{0,1\}$ to be constantly 1 on $(\omega \backslash n) \times \omega$ and to be 0 otherwise. Then $X \cup\left\{y_{i}: i<\right.$ $n\} \cup\left\{v_{n}\right\}$ is a maximal orthogonal family. The fact that every orthogonal family extending $X$ is countable follows easily from noting that if $u, v$ are nontrivial scalar multiples of $(1,1,1, \ldots)$, then $u$ and $v$ cannot be orthogonal. This proves part (a).

To prove part (b) we construct a perfect tree $T \subseteq \mathbb{R}^{<\omega}$ as follows. Let $T_{0}=\{()\}, T_{1}=\{(1)\}$ and $T_{2}=\{(1,1),(1,-1)\}$. We will do the construction of Kunen (Theorem 2) except we will use unequal size weights at each stage. Each $T_{n} \subseteq \mathbb{R}^{n}$ will consist of $n$ pairwise orthogonal elements of $\mathbb{R}^{n}$. The set $T_{n+1}$ will be obtained by picking exactly one element $s_{n}$ of $T_{n}$ and a pair $\delta_{n}, b_{n}>0$ with the property that $\left(s_{n}, s_{n}\right)=\delta_{n} \cdot b_{n}$ and then letting

$$
T_{n+1}=\left\{t^{\wedge} 0: t \in T_{n}, t \neq s_{n}\right\} \cup\left\{s_{n}{ }^{\wedge} \delta_{n}, s_{n}{ }^{\wedge}-b_{n}\right\} .
$$

Taking $P=\left\{x \in \mathbb{R}^{\omega}: \forall n x \uparrow n \in T_{n}\right\}$, the $s_{n}$ are chosen so that $P$ has no isolated points and hence is perfect. The only remaining things to be picked are the $\delta_{n}$ and $b_{n}$. Let $\delta_{1}=b_{1}=1$.

Given $T_{n} \subseteq \mathbb{R}^{n}$ for $n \geq 2$ let

$$
m=\min \left\{\max \left\{|(s, x)|: s \in T_{n}\right\}: x \in \mathbb{R}^{n}, \frac{3}{4} \leq\|x\|_{2} \leq 1\right\} .
$$

Here $(s, x)$ refers to the ordinary inner product in $\mathbb{R}^{n}$ and $\|x\|_{2}$ the $l_{2}$ norm of $x$. By compactness it is clear that $m>0$, so we can let

$$
\delta_{n}=\frac{1}{2} \cdot \min \left\{\frac{m}{2}, \delta_{n-1}\right\}
$$


and then choose $b_{n}$ so that $\left(s_{n}, s_{n}\right)=\delta_{n} \cdot b_{n}$.

Just as in the proof of Theorem 2 the family $P$ is a maximal orthogonal family in $\mathbb{R}^{\omega}$. Now define $E \subseteq P$ as follows:

$$
E=\left\{x \in P: \exists n \forall m>n\left(x(m) \in\left\{0, \delta_{m}\right\}\right)\right\} .
$$

Since $\delta_{m+1} \leq \frac{1}{2^{m}}$, it is clear that $E \subseteq l_{2}$. We claim $E$ is a maximal orthogonal family in $l_{2}$. Suppose $x \in l_{2}$ is nontrivial and by taking a scalar multiple (if necessary) assume $\|x\|_{2}=1$. We will find an element of $E$ which has nonzero inner product with $x$. Choose $n$ sufficiently large so that

$$
\|x \uparrow n\|_{2} \geq \frac{3}{4}
$$

Choose $s \in T_{n}$ so that $\delta_{n} \leq \frac{1}{4}|(s, x \uparrow n)|$. Let $y \in E$ be defined by $y\lceil n=s$ and $y(m) \in\left\{0, \delta_{m}\right\}$ for all $m \geq n$. Note that since $\|x\|_{2}=1,|x(m)| \leq 1$ and thus by our choice of $\delta_{m}$ 's $\left(\delta_{m} \leq \frac{1}{2^{m-n}} \delta_{m}\right)$

$$
\mid\left(x \uparrow n, y\lceil n)\left|>\sum_{m \geq n}\right| x(m) y(m) \mid\right.
$$

and consequently $(x, y) \neq 0$. This proves Theorem $\square$.

\section{Open Questions.}

1. (Abian) Does there exists a model of ZFC with no maximal orthogonal family of cardinality $\omega_{1}$ ? In particular, what happens under MA or PFA?

2. Is it always the case that for any uncountable $\kappa$ there is a maximal orthogonal family of cardinality $\kappa$ iff there exists a maximal almost disjoint family of subsets of $\omega$ of cardinality $\kappa$ ?

3. (Kunen) If there is a maximal orthogonal family of cardinality $\kappa$, then does there exists one of cardinality $\kappa$ with almost disjoint supports? 


\section{References}

[1] S.Hechler, Short complete nested sequences in $\beta N \backslash N$ and small maximal almost-disjoint families, General Topology and Its Applications, 2(1972), 139-149.

[2] K.Kunen, Set Theory, North-Holland 1980, Thm 2.3 p 256.

[3] A.Miller, Infinite combinatorics and definability, Annals of Pure and Applied Mathematical Logic, 41(1989), 179-203,

[4] J.R.Retherford, Hilbert space: Compact Operators and the Trace Theorem, London Mathematical Society Student Texts, 21(1993), Cambridge University Press.

Addresses

Arnold W. Miller

University of Wisconsin-Madison

Department of Mathematics Van Vleck Hall

480 Lincoln Drive

Madison, Wisconsin 53706-1388, USA

e-mail: miller@math.wisc.edu

Juris Steprans

York University

Department of Mathematics

North York, Ontario M3J 1P3, Canada

e-mail: juris.steprans@mathstat.yorku.ca

July 1995. 